\title{
Refinements of Generalized Aczél's Inequality and Bellman's Inequality and Their Applications
}

\author{
Jing-Feng Tian' ${ }^{1}$ and Shu-Yan Wang ${ }^{2}$ \\ ${ }^{1}$ College of Science and Technology, North China Electric Power University, Baoding, Hebei Province 071051, China \\ ${ }^{2}$ Department of Mathematics and Computer, Baoding University, Baoding, Hebei Province 071000, China
}

Correspondence should be addressed to Jing-Feng Tian; tianjfhxm_ncepu@yahoo.cn

Received 13 October 2012; Accepted 30 December 2012

Academic Editor: Shanhe Wu

Copyright (c) 2013 J.-F. Tian and S.-Y. Wang. This is an open access article distributed under the Creative Commons Attribution License, which permits unrestricted use, distribution, and reproduction in any medium, provided the original work is properly cited.

We give some refinements of generalized Aczél's inequality and Bellman's inequality proposed by Tian. As applications, some refinements of integral type of generalized Aczél's inequality and Bellman's inequality are given.

\section{Introduction}

The famous Aczél's inequality [1] states that if $a_{i}, b_{i}(i=$ $1,2, \ldots, n)$ are real numbers such that $a_{1}^{2}-\sum_{i=2}^{n} a_{i}^{2}>0$ and $b_{1}^{2}-\sum_{i=2}^{n} b_{i}^{2}>0$, then

$$
\left(a_{1}^{2}-\sum_{i=2}^{n} a_{i}^{2}\right)\left(b_{1}^{2}-\sum_{i=2}^{n} b_{i}^{2}\right) \leq\left(a_{1} b_{1}-\sum_{i=2}^{n} a_{i} b_{i}\right)^{2} .
$$

It is well known that Aczél's inequality plays an important role in the theory of functional equations in non-Euclidean geometry. In recent years, various attempts have been made by many authors to improve and generalize the Aczél's inequality (see [2-19] and references therein). We state here some improvements of Aczél's inequality.

One of the most important results in the references mentioned above is an exponential extension of (1), which is stated in the following theorem.

Theorem A. Let $p$ and $q$ be real numbers such that $p, q \neq 0$, and $1 / p+1 / q=1$ and let $a_{i}, b_{i}(i=1,2, \ldots, n)$ be positive numbers such that $a_{1}^{p}-\sum_{i=2}^{n} a_{i}^{p}>0$ and $b_{1}^{q}-\sum_{i=2}^{n} b_{i}^{q}>0$. If $p>1$, then

$$
\left(a_{1}^{p}-\sum_{i=2}^{n} a_{i}^{p}\right)^{1 / p}\left(b_{1}^{q}-\sum_{i=2}^{n} b_{i}^{q}\right)^{1 / q} \leq a_{1} b_{1}-\sum_{i=2}^{n} a_{i} b_{i} .
$$

If $p<1(p \neq 0)$, then the reverse inequality in (2) holds.
Remark 1. The case $p>1$ of Theorem A was proved by Popoviciu [8]. The case $p<1$ was given in [15] by Vasić and Pečarić.

Vasić and Pečarić [16] presented a further extension of inequality (1).

Theorem B. Let $a_{r j}>0, \lambda_{j}>0, a_{1 j}^{\lambda_{j}}-\sum_{r=2}^{n} a_{r j}^{\lambda_{j}}>0, r=$ $1,2, \ldots, n, j=1,2, \ldots, m$, and let $\sum_{j=1}^{m}\left(1 / \lambda_{j}\right) \geq 1$. Then

$$
\prod_{j=1}^{m}\left(a_{1 j}^{\lambda_{j}}-\sum_{r=2}^{n} a_{r j}^{\lambda_{j}}\right)^{1 / \lambda_{j}} \leq \prod_{j=1}^{m} a_{1 j}-\sum_{r=2}^{n} \prod_{j=1}^{m} a_{r j} .
$$

In a recent paper [18], Wu and Debnath established an interesting generalization of Aczél's inequality [1] as follows.

Theorem C. Let $a_{r j}>0, \lambda_{j}>0, a_{1 j}^{\lambda_{j}}-\sum_{r=2}^{n} a_{r j}^{\lambda_{j}}>0, r=$ $1,2, \ldots, n, j=1,2, \ldots, m$, and let $\rho=\min \left\{\sum_{j=1}^{m}\left(1 / \lambda_{j}\right), 1\right\}$. Then

$$
\prod_{j=1}^{m}\left(a_{1 j}^{\lambda_{j}}-\sum_{r=2}^{n} a_{r j}^{\lambda_{j}}\right)^{1 / \lambda_{j}} \leq n^{1-\rho} \prod_{j=1}^{m} a_{1 j}-\sum_{r=2}^{n} \prod_{j=1}^{m} a_{r j} .
$$

In 2012, Tian [10] presented the following reversed version of inequality (4). 
Theorem D. Let $a_{r j}>0, \lambda_{1} \neq 0, \lambda_{j}<0(j=2,3, \ldots, m)$, $a_{1 j}^{\lambda_{j}}-\sum_{r=2}^{n} a_{r j}^{\lambda_{j}}>0, r=1,2, \ldots, n, j=1,2, \ldots, m$, and let $\tau=\max \left\{\sum_{j=1}^{m}\left(1 / \lambda_{j}\right), 1\right\}$. Then

$$
\prod_{j=1}^{m}\left(a_{1 j}^{\lambda_{j}}-\sum_{r=2}^{n} a_{r j}^{\lambda_{j}}\right)^{1 / \lambda_{j}} \geq n^{1-\tau} \prod_{j=1}^{m} a_{1 j}-\sum_{r=2}^{n} \prod_{j=1}^{m} a_{r j}
$$

Therefore, applying the above inequality, Tian gave the reversed version of inequality (3) as follows.

Theorem E. Let $a_{r j}>0, \lambda_{1} \neq 0, \lambda_{j}<0(j=2,3, \ldots, m)$, $\sum_{j=1}^{m}\left(1 / \lambda_{j}\right) \leq 1, a_{1 j}^{\lambda_{j}}-\sum_{r=2}^{n} a_{r j}^{\lambda_{j}}>0$, and $r=1,2, \ldots, n, j=$ $1,2, \ldots, m$. Then

$$
\prod_{j=1}^{m}\left(a_{1 j}^{\lambda_{j}}-\sum_{r=2}^{n} a_{r j}^{\lambda_{j}}\right)^{1 / \lambda_{j}} \geq \prod_{j=1}^{m} a_{1 j}-\sum_{r=2}^{n} \prod_{j=1}^{m} a_{r j}
$$

Moreover, in [10], Tian obtained the following integral form of inequality (5).

Theorem F. Let $\lambda_{1}>0, \lambda_{j}<0(j=2,3, \ldots, m), \sum_{j=1}^{m} \lambda_{j}=1$, $P_{j}>0(j=1,2, \ldots, m)$, and let $f_{j}(x)(j=1,2, \ldots, m)$ be positive Riemann integrable functions on $[a, b]$ such that $P_{j}^{\lambda_{j}}-$ $\int_{a}^{b} f_{j}^{\lambda_{j}}(x) \mathrm{d} x>0$. Then

$$
\prod_{j=1}^{m}\left(P_{j}^{\lambda_{j}}-\int_{a}^{b} f_{j}^{\lambda_{j}}(x) \mathrm{d} x\right)^{1 / \lambda_{j}} \geq \prod_{j=1}^{m} P_{j}-\int_{a}^{b} \prod_{j=1}^{m} f_{j}(x) \mathrm{d} x .
$$

Bellman inequality [20] related with Aczél's inequality is stated as follows.

Theorem G. Let $a_{i}, b_{i}(i=1,2, \ldots, n)$ be positive numbers such that $a_{1}^{p}-\sum_{i=2}^{n} a_{i}^{p}>0$ and $b_{1}^{q}-\sum_{i=2}^{n} b_{i}^{q}>0$. If $p \geq 1$, then

$$
\begin{gathered}
{\left[\left(a_{1}^{p}-\sum_{i=2}^{n} a_{i}^{p}\right)^{1 / p}+\left(b_{1}^{p}-\sum_{i=2}^{n} b_{i}^{p}\right)^{1 / p}\right]^{p}} \\
\leq\left(a_{1}+b_{1}\right)^{p}-\sum_{i=2}^{n}\left(a_{i}+b_{i}\right)^{p}
\end{gathered}
$$

If $0<p<1$, then the reverse inequality in (8) holds.

Remark 2. The case $p>1$ of Theorem $G$ was proposed by Bellman [20]. The case $0<p<1$ was proved in [15] by Vasić and Pečarić.

The main purpose of this work is to give refinements of inequalities (5) and (8). As applications, some refinements of integral type of inequality (5) and (8) are given.

\section{Refinements of Generalized Aczél's Inequality and Bellman's Inequality}

Theorem 3. Let $a_{r j}>0, \lambda_{1} \neq 0, \lambda_{j}<0(j=2,3, \ldots, m)$, $a_{1 j}^{\lambda_{j}}-\sum_{r=2}^{n} a_{r j}^{\lambda_{j}}>0, r=1,2, \ldots, n, j=1,2, \ldots, m$, and let $\tau=\max \left\{\sum_{j=1}^{m}\left(1 / \lambda_{j}\right), 1\right\}$. Then

$$
\prod_{j=1}^{m}\left(a_{1 j}^{\lambda_{j}}-\sum_{r=2}^{n} a_{r j}^{\lambda_{j}}\right)^{1 / \lambda_{j}} \geq T\left(s, a_{i j}\right) \geq n^{1-\tau} \prod_{j=1}^{m} a_{1 j}-\sum_{r=2}^{n} \prod_{j=1}^{m} a_{r j},
$$

where

$$
T\left(s, a_{i j}\right)=(n+1-s)^{1-\tau} \prod_{j=1}^{m}\left(a_{1 j}^{\lambda_{j}}-\sum_{r=2}^{s} a_{r j}^{\lambda_{j}}\right)^{1 / \lambda_{j}}-\sum_{r=s+1}^{n} \prod_{j=1}^{m} a_{r j} .
$$

Proof

Case (I). When $\sum_{j=1}^{m}\left(1 / \lambda_{j}\right)<1$, then $\tau=1$. On the one hand, we split the left-hand side of inequality (9) as follows:

$$
\begin{aligned}
\prod_{j=1}^{m}\left(a_{1 j}^{\lambda_{j}}-\sum_{r=2}^{n} a_{r j}^{\lambda_{j}}\right)^{1 / \lambda_{j}} & =\prod_{j=1}^{m}\left(a_{1 j}^{\lambda_{j}}-\sum_{r=2}^{s} a_{r j}^{\lambda_{j}}-\sum_{r=s+1}^{n} a_{r j}^{\lambda_{j}}\right)^{1 / \lambda_{j}} \\
& =\prod_{j=1}^{m}\left(A_{j}^{\lambda_{j}}-\sum_{r=s+1}^{n} a_{r j}^{\lambda_{j}}\right)^{1 / \lambda_{j}}
\end{aligned}
$$

where

$$
A_{j}=\left(a_{1 j}^{\lambda_{j}}-\sum_{r=2}^{s} a_{r j}^{\lambda_{j}}\right)^{1 / \lambda_{j}}, \quad j=1,2, \ldots, m .
$$

From this hypothesis, it is immediate to obtain the inequality

$$
A_{j}^{\lambda_{j}} \geq \sum_{r=s+1}^{n} a_{r j}^{\lambda_{j}}
$$

Thus, by using inequality (6), we have

$$
\prod_{j=1}^{m}\left(A_{j}^{\lambda_{j}}-\sum_{r=s+1}^{n} a_{r j}^{\lambda_{j}}\right)^{1 / \lambda_{j}} \geq \prod_{j=1}^{m} A_{j}-\sum_{r=s+1}^{n} \prod_{j=1}^{m} a_{r j} .
$$

On the other hand, by using inequality (6) again, we obtain the inequality

$$
\prod_{j=1}^{m} A_{j}=\prod_{j=1}^{m}\left(a_{1 j}^{\lambda_{j}}-\sum_{r=2}^{s} a_{r j}^{\lambda_{j}}\right)^{1 / \lambda_{j}} \geq \prod_{j=1}^{m} a_{1 j}-\sum_{r=2}^{s} \prod_{j=1}^{m} a_{r j} .
$$

Combining inequalities (11), (14), and (15) we can get inequality (9).

Case (II). When $\sum_{j=1}^{m}\left(1 / \lambda_{j}\right) \geq 1$, using Case (I) with $\sum_{j=1}^{m+1}\left(1 / \lambda_{j}\right)=1$, we have

$\prod_{j=1}^{m+1}\left(a_{1 j}^{\lambda_{j}}-\sum_{r=2}^{n} a_{r j}^{\lambda_{j}}\right)^{1 / \lambda_{j}} \geq \prod_{j=1}^{m+1}\left(a_{1 j}^{\lambda_{j}}-\sum_{r=2}^{s} a_{r j}^{\lambda_{j}}\right)^{1 / \lambda_{j}}-\sum_{r=s+1}^{n} \prod_{j=1}^{m+1} a_{r j}$, 
where $a_{1 j}^{\lambda_{j}}-\sum_{r=2}^{n} a_{r j}^{\lambda_{j}}>0, a_{r j}>0, \lambda_{1} \neq 0, \lambda_{j}<0(j=$ $2,3, \ldots, m+1)$.

Hence, taking $a_{1(m+1)}^{\lambda_{m+1}}=1, a_{2(m+1)}^{\lambda_{m+1}}=a_{3(m+1)}^{\lambda_{m+1}}=\cdots=$ $a_{n(m+1)}^{\lambda_{m+1}}=1 / n$ into (16), we obtain

$$
\begin{aligned}
\left(\frac{1}{n}\right)^{1-1 / \lambda_{1}-1 / \lambda_{2}-\cdots-1 / \lambda_{m}} \prod_{j=1}^{m}\left(a_{1 j}^{\lambda_{j}}-\sum_{r=2}^{n} a_{r j}^{\lambda_{j}}\right)^{1 / \lambda_{j}} \\
\geq\left(\frac{n+1-s}{n}\right)^{1-1 / \lambda_{1}-1 / \lambda_{2}-\cdots-1 / \lambda_{m}} \\
\quad \times \prod_{j=1}^{m}\left(a_{1 j}^{\lambda_{j}}-\sum_{r=2}^{s} a_{r j}^{\lambda_{j}}\right)^{1 / \lambda_{j}} \\
-\left(\frac{1}{n}\right)^{1-1 / \lambda_{1}-1 / \lambda_{2}-\cdots-1 / \lambda_{m}} \sum_{r=s+1}^{n} \prod_{j=1}^{m} a_{r j},
\end{aligned}
$$

that is,

$$
\begin{aligned}
\prod_{j=1}^{m}\left(a_{1 j}^{\lambda_{j}}-\sum_{r=2}^{n} a_{r j}^{\lambda_{j}}\right)^{1 / \lambda_{j}} \geq & (n+1-s)^{1-1 / \lambda_{1}-1 / \lambda_{2}-\cdots-1 / \lambda_{m}} \\
& \times \prod_{j=1}^{m}\left(a_{1 j}^{\lambda_{j}}-\sum_{r=2}^{s} a_{r j}^{\lambda_{j}}\right)^{1 / \lambda_{j}} \\
& -\sum_{r=s+1}^{n} \prod_{j=1}^{m} a_{r j} .
\end{aligned}
$$

Therefore, repeating the foregoing arguments, we get

$$
\begin{gathered}
(n+1-s)^{1-1 / \lambda_{1}-1 / \lambda_{2}-\cdots-1 / \lambda_{m}} \prod_{j=1}^{m}\left(a_{1 j}^{\lambda_{j}}-\sum_{r=2}^{s} a_{r j}^{\lambda_{j}}\right)^{1 / \lambda_{j}} \\
-\sum_{r=s+1}^{n} \prod_{j=1}^{m} a_{r j} \geq n^{1-\tau} \prod_{j=1}^{m} a_{1 j}-\sum_{r=2}^{n} \prod_{j=1}^{m} a_{r j} .
\end{gathered}
$$

Combining inequalities (18) and (19) leads to inequality (9) immediately. The proof of Theorem 3 is completed.

If we set $\sum_{j=1}^{m}\left(1 / \lambda_{j}\right) \leq 1$, then from Theorem 3, we obtain the following refinement of inequality (6).

Corollary 4. Let $a_{r j}>0, \lambda_{1} \neq 0, \lambda_{j}<0(j=2,3, \ldots, m)$, $\sum_{j=1}^{m}\left(1 / \lambda_{j}\right) \leq 1, a_{1 j}^{\lambda_{j}}-\sum_{r=2}^{n} a_{r j}^{\lambda_{j}}>0, r=1,2, \ldots, n$, and $j=$ $1,2, \ldots, m$. Then

$$
\begin{aligned}
\prod_{j=1}^{m}\left(a_{1 j}^{\lambda_{j}}-\sum_{r=2}^{n} a_{r j}^{\lambda_{j}}\right)^{1 / \lambda_{j}} & \geq \prod_{j=1}^{m}\left(a_{1 j}^{\lambda_{j}}-\sum_{r=2}^{s} a_{r j}^{\lambda_{j}}\right)^{1 / \lambda_{j}}-\sum_{r=s+1}^{n} \prod_{j=1}^{m} a_{r j} \\
& \geq \prod_{j=1}^{m} a_{1 j}-\sum_{r=2}^{n} \prod_{j=1}^{m} a_{r j} .
\end{aligned}
$$

Putting $m=2, \lambda_{1}=p \neq 0, \lambda_{2}=q<0, a_{r 1}=a_{r}$, and $a_{r 2}=$ $b_{r}(r=1,2, \ldots, n)$ in Theorem 3, we obtain the refinement and generalization of Theorem A for $p<1$.
Corollary 5. Let $a_{r}>0, b_{r}>0(r=1,2, \ldots, n), a_{1}^{p}-$ $\sum_{r=2}^{n} a_{r}^{p}>0, b_{1}^{q}-\sum_{r=2}^{n} b_{r}^{q}>0, p \neq 0, q<0$, and $\rho=$ $\max \{1 / p+1 / q, 1\}$. Then, the following inequality holds:

$$
\begin{aligned}
\left(a_{1}^{p}-\sum_{r=2}^{n} a_{r}^{p}\right)^{1 / p}\left(b_{1}^{q}-\sum_{r=2}^{n} b_{r}^{q}\right)^{1 / q} \\
\geq(n+1-s)^{1-\rho}\left(a_{1}^{p}-\sum_{r=2}^{s} a_{r}^{p}\right)^{1 / p} \\
\quad \times\left(b_{1}^{q}-\sum_{r=2}^{s} b_{r}^{q}\right)^{1 / q}-\sum_{r=s+1}^{n} a_{r} b_{r} \\
\geq n^{1-\rho} a_{1} b_{1}-\sum_{r=2}^{n} a_{r} b_{r} .
\end{aligned}
$$

Based on the mathematical induction, it is easy to see that the following generalized Bellman's inequality is true.

Theorem 6. Let $a_{r j}>0, a_{1 j}^{\lambda_{j}}-\sum_{r=2}^{n} a_{r j}^{\lambda_{j}}>0, r=1,2, \ldots, n$, $j=1,2, \ldots, m$, and let $0<p<1$. Then

$$
\left[\prod_{j=1}^{m}\left(a_{1 j}^{p}-\sum_{r=2}^{n} a_{r j}^{p}\right)^{1 / p}\right]^{p} \geq\left(\sum_{j=1}^{m} a_{1 j}\right)^{p}-\sum_{r=2}^{n}\left(\sum_{j=1}^{m} a_{r j}\right)^{p} .
$$

Next, we give a refinement of generalized Bellman's inequality (22) as follows.

Theorem 7. Let $a_{r j}>0, a_{1 j}^{\lambda_{j}}-\sum_{r=2}^{n} a_{r j}^{\lambda_{j}}>0, r=1,2, \ldots, n$, $j=1,2, \ldots, m$, and let $0<p<1$. Then

$$
\begin{aligned}
{\left[\prod_{j=1}^{m}\left(a_{1 j}^{p}-\sum_{r=2}^{n} a_{r j}^{p}\right)^{1 / p}\right]^{p} } & \geq\left[\prod_{j=1}^{m}\left(a_{1 j}^{p}-\sum_{r=2}^{s} a_{r j}^{p}\right)^{1 / p}\right]^{p} \\
& -\sum_{r=s+1}^{n}\left(\sum_{j=1}^{m} a_{r j}\right)^{p} \\
\geq & \left(\sum_{j=1}^{m} a_{1 j}\right)^{p}-\sum_{r=2}^{n}\left(\sum_{j=1}^{m} a_{r j}\right)^{p} .
\end{aligned}
$$

Proof. The proof of Theorem 7 is similar to the one of Theorem 3. Applying generalized Bellman's inequality (22) twice, we can deduce the inequality (23).

\section{Application}

In this section, we show two applications of the inequalities newly obtained in Section 2. 
Firstly, we give an improvement of inequality (7) by using Theorem 3.

Theorem 8. Let $B_{j}>0(j=1,2, \ldots, m)$, let $\lambda_{1}>0$, $\lambda_{j}<0(j=2,3, \ldots, m), \sum_{j=1}^{m} \lambda_{j}=1$, and let $f_{j}(x)(j=$ $1,2, \ldots, m)$ be positive integrable functions defined on $[a, b]$ with $B_{j}^{\lambda_{j}}-\int_{a}^{b} f_{j}^{\lambda_{j}}(x) \mathrm{d} x>0$. Then, for any $t \in[a, b)$, one has

$$
\begin{aligned}
& \prod_{j=1}^{m}\left(B_{j}^{\lambda_{j}}-\int_{a}^{b} f_{j}^{\lambda_{j}}(x) \mathrm{d} x\right)^{1 / \lambda_{j}} \\
& \quad \geq \prod_{j=1}^{m}\left(B_{j}^{\lambda_{j}}-\int_{a}^{t} f_{j}^{\lambda_{j}}(x) \mathrm{d} x\right)^{1 / \lambda_{j}}-\int_{t}^{b} \prod_{j=1}^{m} f_{j}(x) \mathrm{d} x \\
& \quad \geq \prod_{j=1}^{m} B_{j}-\int_{a}^{b} \prod_{j=1}^{m} f_{j}(x) \mathrm{d} x .
\end{aligned}
$$

Proof. We need to prove only the left side of inequality (24). The proof of the right side of inequality (24) is similar. For any positive integers $n$ and $l$, we choose an equidistant partition of $[a, t]$ and $[t, b]$, respectively, as

$$
\begin{gathered}
a<a+\frac{c-a}{n}<\cdots<a+\frac{c-a}{n} k \\
<\cdots<a+\frac{c-a}{n}(n-1)<c, \\
c<c+\frac{b-c}{l}<\cdots<c+\frac{b-c}{l} i \\
<\cdots<c+\frac{b-c}{l}(l-1)<b, \\
x_{k}=a+\frac{c-a}{n} k, \quad \Delta x_{k}=\frac{c-a}{n}, \quad k=1,2, \ldots, n, \\
x_{i}=c+\frac{b-c}{l} i, \quad \Delta x_{i}=\frac{b-c}{l}, \quad i=1,2, \ldots, n .
\end{gathered}
$$

Noting that $B_{j}^{\lambda_{j}}-\int_{a}^{b} f_{j}^{\lambda_{j}}(x) \mathrm{d} x=B_{j}^{\lambda_{j}}-\left(\int_{a}^{c} f_{j}^{\lambda_{j}}(x) \mathrm{d} x+\right.$ $\left.\int_{c}^{b} f_{j}^{\lambda_{j}}(x) \mathrm{d} x\right)>0(j=1,2, \ldots, m)$, we have

$$
\begin{array}{r}
B_{j}^{\lambda_{j}}-\left\{\lim _{n \rightarrow \infty} \sum_{k=1}^{n} f_{j}^{\lambda_{j}}\left[a+\frac{k(c-a)}{n}\right] \frac{c-a}{n}\right. \\
\left.+\lim _{l \rightarrow \infty} \sum_{i=1}^{l} f_{j}^{\lambda_{j}}\left[c+\frac{i(b-c)}{l}\right] \frac{b-c}{l}\right\}>0, \\
\quad(j=1,2, \ldots, m) .
\end{array}
$$

Consequently, there exists a positive integer $N$, such that

$$
\begin{aligned}
B_{j}^{\lambda_{j}}-\left[\sum_{k=1}^{n} f_{j}^{\lambda_{j}}\left(a+\frac{k(c-a)}{n}\right) \frac{c-a}{n}\right. \\
\left.+\sum_{i=1}^{l} f_{j}^{\lambda_{j}}\left(c+\frac{i(b-c)}{l}\right) \frac{b-c}{l}\right]>0
\end{aligned}
$$

for all $n, l>N$ and $j=1,2, \ldots, m$.

By using Theorem 3, for any $n, l>N$, the following inequality holds:

$$
\begin{aligned}
\prod_{j=1}^{m}\left\{B_{j}^{\lambda_{j}}-\right. & {\left[\sum_{k=1}^{n} f_{j}^{\lambda_{j}}\left(a+\frac{k(c-a)}{n}\right) \frac{c-a}{n}\right.} \\
& \left.\left.+\sum_{i=1}^{l} f_{j}^{\lambda_{j}}\left(c+\frac{i(b-c)}{l}\right) \frac{b-c}{l}\right]\right\}^{1 / \lambda_{j}} \\
\geq & \prod_{j=1}^{m}\left[B_{j}^{\lambda_{j}}-\sum_{k=1}^{n} f_{j}^{\lambda_{j}}\left(a+\frac{k(c-a)}{n}\right) \frac{c-a}{n}\right]^{1 / \lambda_{j}} \\
& -\sum_{i=1}^{l}\left[\prod_{j=1}^{m} f_{j}\left(c+\frac{i(b-c)}{l}\right)\right]\left(\frac{b-c}{l}\right)^{\sum_{j=1}^{m}\left(1 / \lambda_{j}\right)} .
\end{aligned}
$$

Since

$$
\sum_{j=1}^{m} \frac{1}{\lambda_{j}}=1
$$

we have

$$
\begin{gathered}
\prod_{j=1}^{m}\left\{B_{j}^{\lambda_{j}}-\left[\sum_{k=1}^{n} f_{j}^{\lambda_{j}}\left(a+\frac{k(c-a)}{n}\right) \frac{c-a}{n}\right.\right. \\
\left.\left.\quad+\sum_{i=1}^{l} f_{j}^{\lambda_{j}}\left(c+\frac{i(b-c)}{l}\right) \frac{b-c}{l}\right]\right\}^{1 / \lambda_{j}} \\
\geq \prod_{j=1}^{m}\left[B_{j}^{\lambda_{j}}-\sum_{k=1}^{n} f_{j}^{\lambda_{j}}\left(a+\frac{k(c-a)}{n}\right) \frac{c-a}{n}\right]^{1 / \lambda_{j}} \\
\quad-\sum_{i=1}^{l}\left[\prod_{j=1}^{m} f_{j}\left(c+\frac{i(b-c)}{l}\right)\right]\left(\frac{b-c}{l}\right) .
\end{gathered}
$$

Noting that $f_{j}(x)(j=1,2, \ldots, m)$ are positive Riemann integrable functions on $[a, b]$, we know that $\prod_{j=1}^{m} f_{j}(x)$ and $f_{j}^{\lambda_{j}}(x)$ are also integrable on $[a, b]$. Letting $n \rightarrow \infty$ on both sides of inequality (30), we get the left side of inequality (24). The proof of Theorem 8 is completed.

We give here a direct consequence from Theorem 8 . Putting $m=2, \lambda_{1}=p, \lambda_{2}=q, B_{1}=a_{1}, B_{2}=b_{1}, f_{1}=f$, and $f_{2}=g$ in (24), we obtain a special important case as follows. 
Corollary 9. Let $p$ and $q$ be real numbers such that $p>0, q<$ 0 , and $(1 / p)+(1 / q)=1$, let $a_{1}, b_{1}>0$, and let $f, g$ be positive integrable functions defined on $[a, b]$ with $a_{1}^{p}-\int_{a}^{b} f^{p}(x) \mathrm{d} x>0$ and $b_{1}^{q}-\int_{a}^{b} g^{q}(x) \mathrm{d} x>0$. Then, for any $t \in[a, b)$, one has

$$
\begin{aligned}
\left(a_{1}^{p}\right. & \left.-\int_{a}^{b} f^{p}(x) \mathrm{d} x\right)^{1 / p}\left(b_{1}^{q}-\int_{a}^{b} g^{q}(x) \mathrm{d} x\right)^{1 / q} \\
& \geq\left(a_{1}^{p}-\int_{a}^{c} f^{p}(x) \mathrm{d} x\right)^{1 / p}\left(b_{1}^{q}-\int_{a}^{c} g^{q}(x) \mathrm{d} x\right)^{1 / q} \\
& \geq a_{1} b_{1}-\int_{a}^{b} f(x) g(x) \mathrm{d} x .
\end{aligned}
$$

Finally, we present a refinement of integral type of generalized Bellman's inequality.

Theorem 10. Let $B_{j}>0(j=1,2, \ldots, m)$, let $0<p<1$, and let $f_{j}(x)(j=1,2, \ldots, m)$ be positive integrable functions defined on $[a, b]$ with $B_{j}^{\lambda_{j}}-\int_{a}^{b} f_{j}^{\lambda_{j}}(x) \mathrm{d} x>0$. Then, for any $t \in[a, b)$, one has

$$
\begin{aligned}
& {\left[\sum_{j=1}^{m}\left(B_{j}^{p}-\int_{a}^{b} f_{j}^{p}(x) \mathrm{d} x\right)^{1 / p}\right]^{p}} \\
& \quad \geq\left[\sum_{j=1}^{m}\left(B_{j}^{p}-\int_{a}^{t} f_{j}^{p}(x) \mathrm{d} x\right)^{1 / p}\right]^{p}-\int_{t}^{b}\left(\sum_{j=1}^{m} f_{j}(x)\right)^{p} \mathrm{~d} x \\
& \quad \geq\left(\sum_{j=1}^{m} B_{j}\right)^{p}-\int_{a}^{b}\left(\sum_{j=1}^{m} f_{j}(x)\right)^{p} \mathrm{~d} x .
\end{aligned}
$$

Proof. The proof of Theorem 10 is similar to the proof of Theorem 8.

A special case to the last theorem is as follows.

Corollary 11. Let $0<p<1$, let $a_{1}, b_{1}>0$, and let $f, g$ be positive integrable functions defined on $[a, b]$ with $a_{1}^{p}-$ $\int_{a}^{b} f^{p}(x) \mathrm{d} x>0$ and $b_{1}^{q}-\int_{a}^{b} g^{q}(x) \mathrm{d} x>0$. Then, for any $t \in[a, b)$, one has

$$
\begin{aligned}
& {\left[\left(a_{1}^{p}-\int_{a}^{b} f^{p}(x) \mathrm{d} x\right)^{1 / p}+\left(b_{1}^{p}-\int_{a}^{b} g^{p}(x) \mathrm{d} x\right)^{1 / p}\right]^{p}} \\
& \geq\left[\left(a_{1}^{p}-\int_{a}^{t} f^{p}(x) \mathrm{d} x\right)^{1 / p}+\left(b_{1}^{p}-\int_{a}^{t} g^{p}(x) \mathrm{d} x\right)^{1 / p}\right]^{p} \\
& \quad-\int_{t}^{b}(f(x)+g(x))^{p} \mathrm{~d} x \\
& \geq\left(a_{1}+b_{1}\right)^{p}-\int_{a}^{b}(f(x)+g(x))^{p} \mathrm{~d} x .
\end{aligned}
$$

\section{Acknowledgments}

The authors would like to express hearty thanks to the anonymous referees for their great efforts to improve this paper. This work was supported by the NNSF of China (no. 61073121), the Natural Science Foundation of Hebei Province of China (no. F2012402037), the Natural Science Foundation of Hebei Education Department (no. Q2012046), and the Fundamental Research Funds for the Central Universities (no. 11ML65).

\section{References}

[1] J. Aczél, "Some general methods in the theory of functional equations in one variable. New applications of functional equations," Uspekhi Matematicheskikh Nauk, vol. 11, no. 3, pp. 3-68, 1956 (Russian).

[2] E. F. Beckenbach and R. Bellman, Inequalities, Springer, Berlin, Germany, 1983.

[3] J. L. Díaz-Barrero, M. Grau-Sánchez, and P. G. Popescu, "Refinements of Aczél, Popoviciu and Bellman's inequalities," Computers \& Mathematics with Applications, vol. 56, no. 9, pp. 2356-2359, 2008.

[4] G. Farid, J. Pečarić, and A. Ur Rehman, "On refinements of Aczél, Popoviciu, Bellman's inequalities and related results," Journal of Inequalities and Applications, vol. 2010, Article ID 579567, 17 pages, 2010.

[5] G. H. Hardy, J. E. Littlewood, and G. Pólya, Inequalities, Cambridge University Press, Cambridge, UK, 1952.

[6] D. S. Mitrinović, J. E. Pečarić, and A. M. Fink, Classical and New Inequalities in Analysis, vol. 61, Kluwer Academic, Dordrecht, The Netherlands, 1993.

[7] Y. Ouyang and R. Mesiar, "On the Chebyshev type inequality for seminormed fuzzy integral," Applied Mathematics Letters, vol. 22, no. 12, pp. 1810-1815, 2009.

[8] T. Popoviciu, "On an inequality," Gazeta Matematica şi Fizica A, vol. 11, no. 64, pp. 451-461, 1959 (Romanian).

[9] J. Tian, "Inequalities and mathematical properties of uncertain variables," Fuzzy Optimization and Decision Making, vol. 10, no. 4, pp. 357-368, 2011.

[10] J. Tian, "Reversed version of a generalized Aczél's inequality and its application," Journal of Inequalities and Applications, vol. 2012, article 202, 2012.

[11] J. Tian, "Reversed version of a generalized sharp Hölder's inequality and its applications," Information Sciences, vol. 201, pp. 61-69, 2012.

[12] J. Tian, "Extension of Hu Ke's inequality and its applications," Journal of Inequalities and Applications, vol. 2011, article 77, 2011.

[13] J. Tian, "Property of a Hölder-type inequality and its application," Mathematical Inequalities \& Applications. In press.

[14] J. Tian and X. M. Hu, "A new reversed version of a generalized sharp Hölder's inequalityand its applications," Abstract and Applied Analysis. In press.

[15] P. M. Vasić and J. E. Pečarić, "On the Hölder and some related inequalities," Mathematica, vol. 25, no. 1, pp. 95-103, 1982.

[16] P. M. Vasić and J. E. Pečarić, "On the Jensen inequality for monotone functions," Analele Universitatii din Timişoara, vol. 17, no. 1, pp. 95-104, 1979.

[17] S. Vong, "On a generalization of Aczél's inequality," Applied Mathematics Letters, vol. 24, no. 8, pp. 1301-1307, 2011. 
[18] S. Wu and L. Debnath, "Generalizations of Aczél's inequality and Popoviciu's inequality," Indian Journal of Pure and Applied Mathematics, vol. 36, no. 2, pp. 49-62, 2005.

[19] W. Yang, "Refinements of generalized Aczél-Popoviciu's inequality and Bellman's inequality," Computers \& Mathematics with Applications, vol. 59, no. 11, pp. 3570-3577, 2010.

[20] R. Bellman, "On an inequality concerning an indefinite form," The American Mathematical Monthly, vol. 63, pp. 108-109, 1956. 


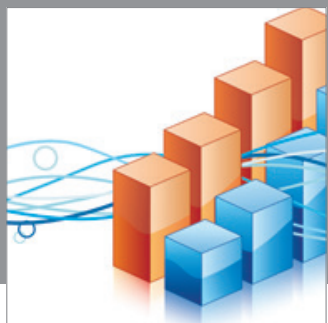

Advances in

Operations Research

mansans

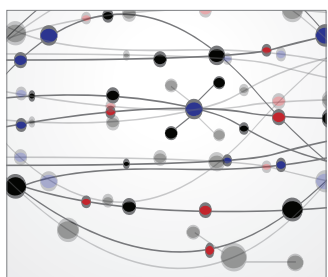

The Scientific World Journal
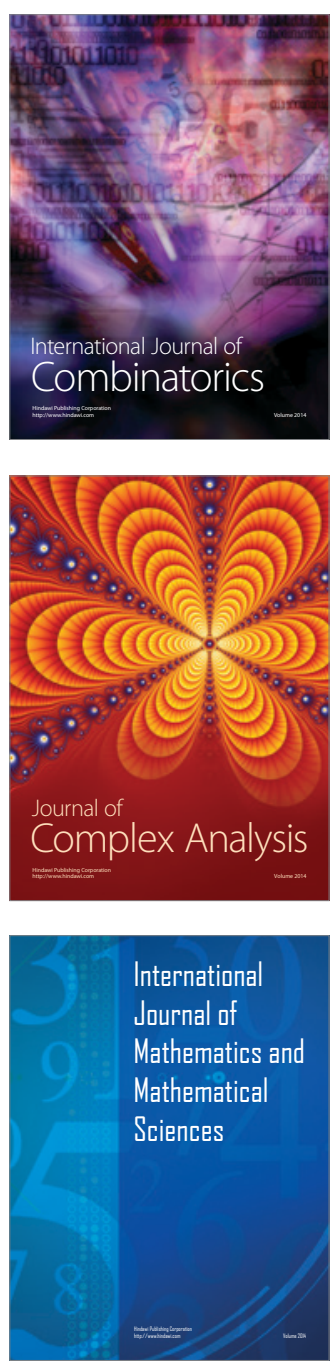
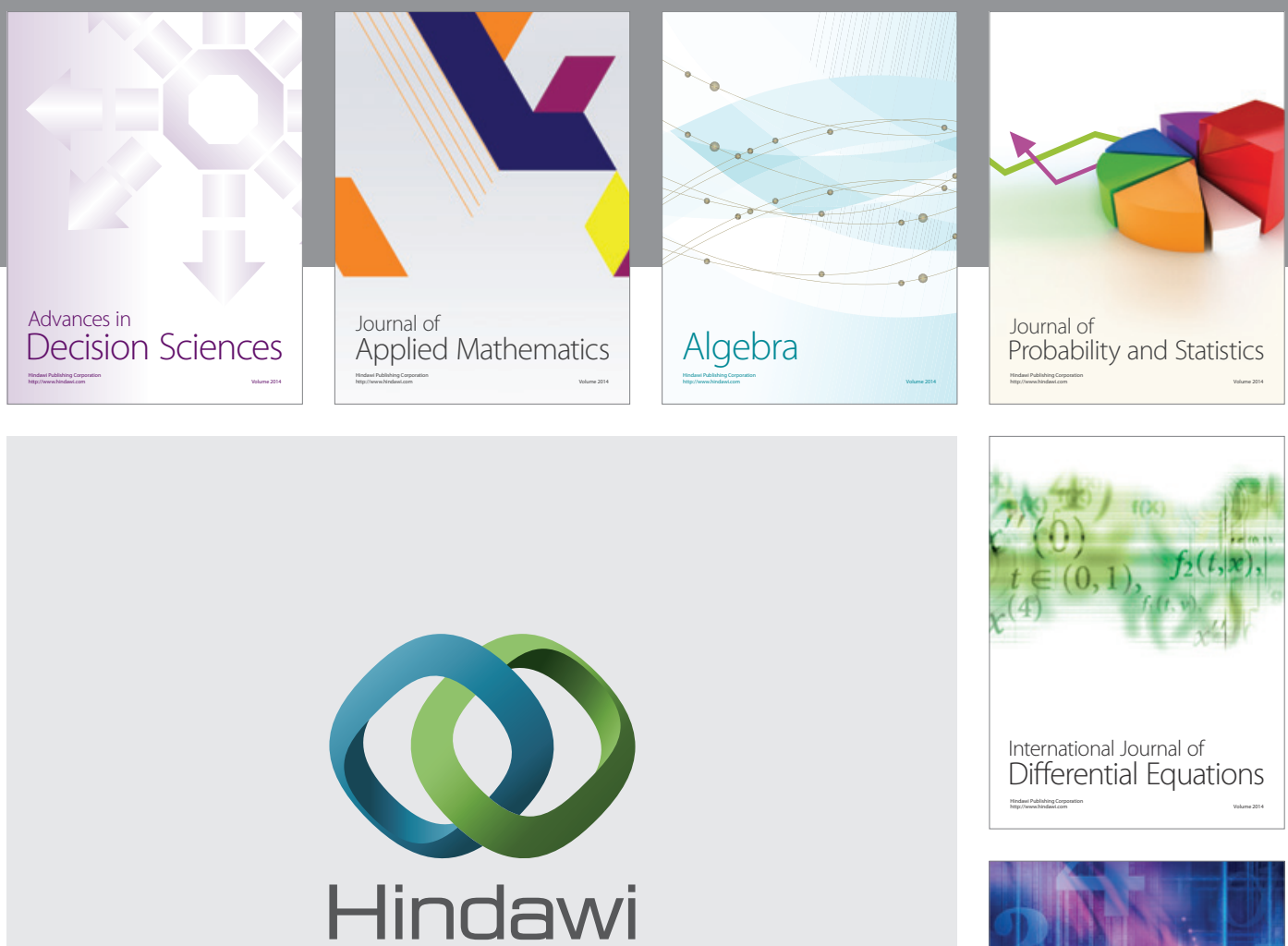

Submit your manuscripts at http://www.hindawi.com
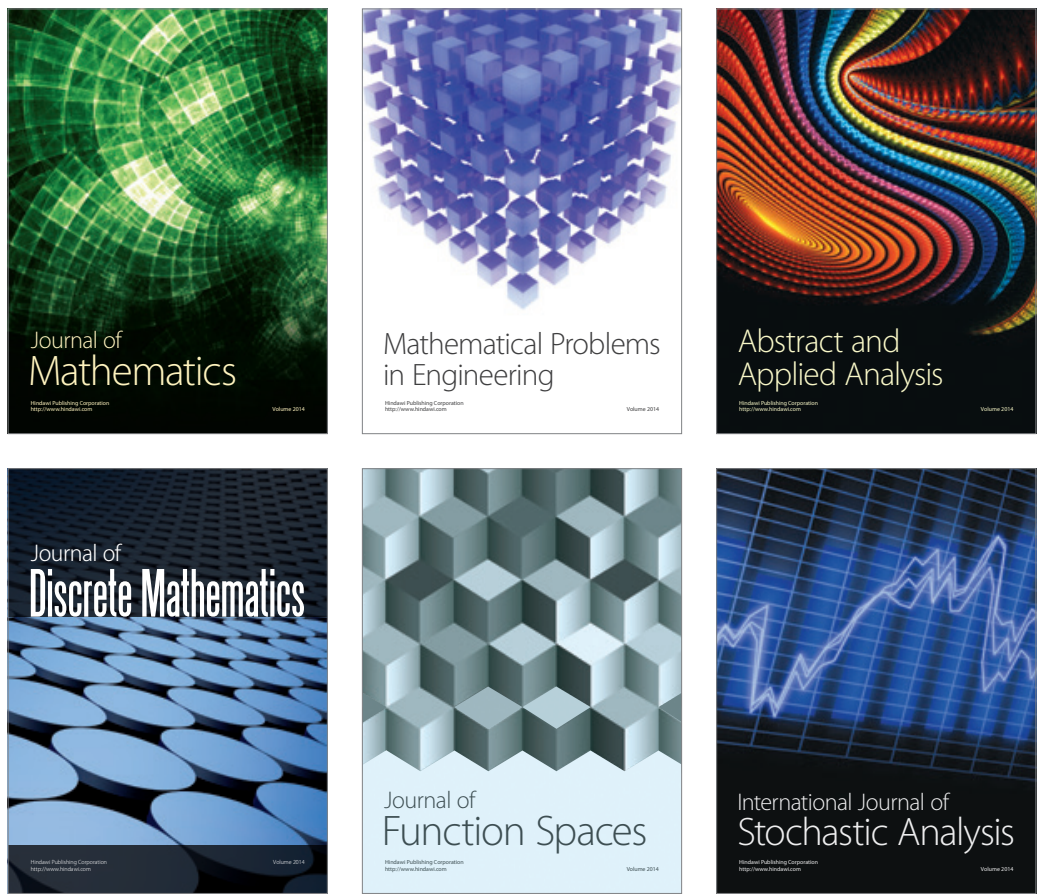

Journal of

Function Spaces

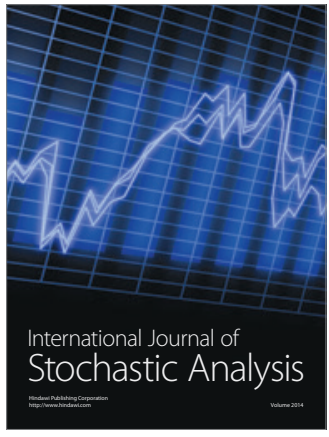

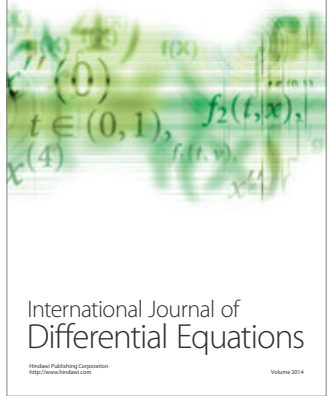
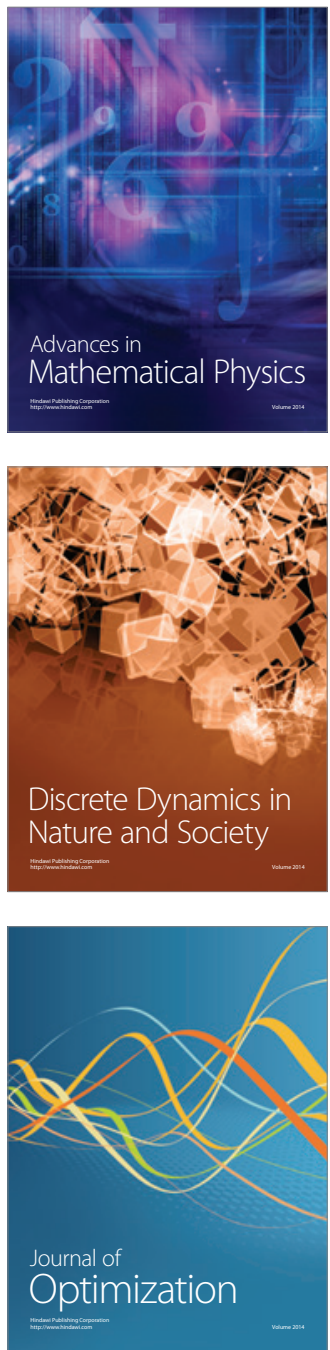